III. Aus der Universitätskinderklinik in Heidelberg. (Director : Prof. Dr. Vierordt.)

\title{
Weitere klinische Untersuchungen über Resorption und Ausscheidung des Kalkes.
}

Von Dr. J. G. Rey in Aachen,

frtherem Assistenten der medicinischen Poliklinik in Heidelberg.

Die Untersuchungen, über welche ich im folgenden zu berichten habe, stellen die Fortführung derjenigen Versuche dar, welche seiner Zeit $R$ üdel hier vorgenommen und über welche derselbe im Archiv für experimentelle Pathologie und Pharmakologie Bd. XXXIII berichtet hat. Ursprünglich waren meine Untersuchungen nur zur weiteren Prüfung der von $R$ üdel erhaltenen Ergebnisse unternommen, sie haben aber ausser der Bestätigung der Angaben jenes Autors noch einiges weitere Bemerkenwerthe zu Tage gefördert, wie sich weiter unten zeigen wird.

Rüdel hat an der Hand einer längeren Versuchsreihe nachgewiesen, dass sich sowohl bei gesunden, als bei rachitischen Kindern durch gleichmässige Ernährung eine gewisse Constanz der Kalkausscheidung im Harn erreichen lässt und dass, wenn hierauf eine grössere Menge anorganischen Kalks per os einverleibt wird, Gesunde wie Rachitische mit einer prompten und erheblichen, rasch vortubergehenden Steigerung der Kalkausscheidung im Harn antworten. Indem Rü del die unter diesen Umständen sehr naheliegende Annahme machte, dass die Menge des Harnkalks einen ungefähren Schluss auf die Gesammtkalkausscheidung und auf die Kalkresorption gestatte, folgerte or aus seinen Versuchen: es könne die Kalkresorption aus dem Darmeanal bei Rachitischen erhebliche Abweichungen von der bei Gesunden nicht zeigen; hieran schloss sich als das bedeutsamste Ergebniss seiner Untersuchungen die weitere bestimmte Folgerung: dass die Rachitis nicht auf mangelhafter Resorption der Kalksalze aus der Nahrung beruhen könne.

Durch andere Versuche zeigte $R \ddot{u} d e l$, wie durch Zufuhr von kalklösenden Säuren $(\mathrm{HCl})$ die Ausscheidung, also aller Wahrscheinkeit nach auch die Resorption der mit der Nahrung eingeführten Kalksalze vermehrt wird, und andererseits, wie durch kalkausfällende Salze '(Natrium phosphoricum) die Kalkausscheidung erheblich vermindert wird. Durch verlangsamte Peristaltik wird 
nach seinen Beobachtungen (Opiumgaben) der Kalk im Urin vermehrt. Durch gesteigerte Peristaltik gelang es ihm nicht, eine deutliche Verminderung zu erreichen, wohl wegen der Kürze der Versuchsanstellung; dagegen beobachtete er bei Durchfällen infolge von Verdauungsstörungen mehrfach ein Sinken des Harnkalks. Einen Unterschied des Kalkgehaltes der Stühle rachitischer und gesunder Kinder konnte Rüdel nicht nachweisen. Der Kalk war im Stuhl in Form schwer löslicher Salze vorhanden. In das Wasserextract gingen nur geringe Mengen $(2-12 \%)$ über. Interessant ist seine Beobachtung, dass mit dem Steigen der Kallkausscheidung im Harn die Menge der in Wasser löslichen Kalksalze in zugehörigen Stuhl abnahm und umgekehrt.

Es ist mir nun zunächst durch Untersuchungen, welche an anderer Stelle veröffentlicht werden, ${ }^{1}$ ) gelungen, ein klares Bild von den Verhältnissen der Kalkausscheidung' als solcher zu gewinnnen; wie bereits bekannt, geht dieselbe nicht nur durch die Nieren, sondern in viel höherem Maasse durch die Schleimhaut des Darms, und zwar des Dickdarms von statten. Dabei scheint das Verhältniss der Ausscheidungsgrösse durch den Darm zu derjenigen durch die Nieren immerhin mit solcher Constanz festgehalten zu werden, dass ein Schluss aus der Harnkalkmenge auf die gesammte ausgeschiedene Kalkmenge und damit auch auf die Kalkresorption erlaubt ist. Damit erhält die oben erwähnte Annahme Rüdel's über die Beziehungen des Harnkalks zur Kalkresorption eine wichtige Stütze. Das Genauere über diese Versuche ist in Jahrgang 1895 des Archivs für experimentelle Pathologie und Pharmakologie nachzusehen.

Ich habe nun weiterhin einige Versuche an rachitischen Kindern zur Prüfung und Verfolgung der Ergebnisse Rüdel's unternommen.

Diese Versuche sind im wesentlichen genau wie diejenigen des genannten Autors angeordnet. Die zum Versuch bestimmten rachitischen Kinder wurden vollständig gleichmässig ernährt; d. h. sie erhielten 14 Tage vor dem Versuch und während der ganzen Dauer des Versuches Tag für Tag qualitativ und quantitativ möglichst dieselbe Nahrung, ein Verfahren, welches für einen Erwachsenen vielleicht unerträglich und schädigend auf die Esslust gewirkt haben würde, welches aber die Kinder nicht nur vertrugen, sondern bei welchem sie auffällig gediehen. Die Kinder waren der Obhut einer zuverlässigen Schwester anvertraut, welche die Aufsammlung des Urins und die Ernährung der Kinder unter meiner eigenen Controlle mit peinlichster Genauigkeit vornahm. Der Urin wurde täglich von Morgens 8 Uhr bis Morgens $8 \mathrm{Uhr}$ aufgefangen. Zum Versuch wurden nur solche Kinder genommen, bei denen die Rachitis vollständig auf der Höhe stand, d.h. wo die Knochen deutliche Auftreibungen, Verkrümmungen etc. zeigten und weich und biegsam waren, also eine Ausheilung noch nicht statthatte. Weiterhin mussten die Kinder bettrein sein und imstande, Urin und Koth getrennt zu entleeren; das ist bei Knaben begreiflicherweise leichter zu erreichen als bei Mädchen; selbst von Knaben, welche den Urin ins Bett lassen, ist es bei grosser Sorgfalt der Pflegerin möglich, sämmtlichen 24 stündigen Urin durch einen vorgebundenen Gummibeutel zu sammeln. Das eine der Versuchskinder war ein Mädchen: dasselbe war indess für sein Alter sehr intelligent und willenskräftig und entleerte Urin und Stuhl in genauester Weise getrennt. - Die Versuche mit Kalkgaben per os wurden nur dann gemacht, wenn der Stuhl des Kindes völlig normal war. Die Kinder nahmen eine Lösung von essigsaurem Kalk, in der sich $8,2 \% \mathrm{CaO}$ fanden, in Sirupus Rubi Idaei recht gern; sie erhielten dieselbe eine Stunde nach der Hauptmahlzeit.

Die Bestimmung des Kalks in dem möglichst genau gewonnenen Tagesurin geschah durch Ausfällen mit oxalsaurem Ammon in essigsaurer Lösung; Filtriren und Glühen, Ueberführen in Gyps, Wägen. In Rechnung wurde der Kalk gebracht als $\mathrm{CaO}$.

Versuch I betraf ein Mädchen, 21/2 Jahre alt, $10,7 \mathrm{~kg}$ schwer; Rachitis.

Status praesens: Fontanelle geschlossen, die Gegend derselben jedoch höckerig, Tubera frontalia sehr stark hervortretend, Zähne zackig und unregelmässig stehend, quergefurcht. Am Thorax nur geringe Zeichen von Rachitis, dagegen sind die Epiphysen der langen Rohrenknochen an Armen und Beinen stark aufgetrieben, die Knochen selbst biegsam.

Das Kind erhielt zunächst durch 14 Tage eine ganz gleichmässige Nahrung, und zwar: 7 Uhr 1 Tasse Milch, 1/2 Semmel; 10 Uhr 1 Tasse Milchcacao, $1 \mathrm{Ei}$; $12 \frac{1}{2}$ Uhr 1 Teller Griesbrei; 4 Uhr 1 Tasse Milch, 1 Semmel; 7 Uhr 1 Tasse Milch, 1/2 Semmel, 1 Ei.

Dann begann der Versuch. Der Urin wurde dabei täglich von Morgens $8 \mathrm{Uhr}$ bis Morgens $8 \mathrm{Uhr}$ aufgefangen. Stuhl und Urin stets getrennt gelassen.

1) Arch. f. experiment. Pathol. u. Pharmakol. Jahrg. 1895.
Die erhaltenen Werthe sind folgende:

\begin{tabular}{l|c|l}
\hline \hline Urinmenge & Kalkmenge CaO & \multicolumn{1}{|c}{ Versuchstag } \\
\hline $600 \mathrm{ccm}$ & 0,0397 & 15. \\
$650 "$ & 0,0418 & $16 .(6 \mathrm{~g}$ kohlensaurer Kalk $)$ \\
$630 "$ & $\mathbf{0 , 0 9 7 5}$ & 17. \\
$650 "$ & $\mathbf{0 , 0 6 2 8}$ & 18. \\
$650 "$ & 0,0427 & $19 .(0,8 \mathrm{~g}$ CaO als essigs. Kalk $)$ \\
$600 "$ & $\mathbf{0 , 0 9 6 1}$ & 20. \\
$800 "$ & $\mathbf{0 , 0 9 3 9}$ & 21. \\
$760 "$ & $\mathbf{0 , 0 8 3 2}$ & 22. \\
$690 "$ & $\mathbf{0 , 0 6 2 6}$ & 23. \\
$650 "$ & 0,0429 & 24.
\end{tabular}

Die Tabelle zeigt deutlich, dass nach einer grossen Gabe kohlensauren Kalks die Vermehrung der Kalkausscheidung eintritt, in den nächsten 24 Stunden nach der Kalkgabe am höchsten ist und am zweiten Tage nachher ebenfalls noch gesteigert ist. Nach einer Gabe von viel weniger essigsaurem Kalk in Lösung wird dieselbe Höhe der Ausscheidung wie vorhin erreicht; dieselbe dauert sogar noch bis zum fünften Tage nach der Kalkgabe allmählich sich vermindernd an. Ebenso fällt es auf, dass die Urinmenge am zweiten Tage nach der Gabe essigsauren Kalks plötzlich erheblich ansteigt.

Es ergiebt sich hiermit eine vollkommene Bestätigung der Rüdel'schen Angaben.

Versuch II. Knabe, 2/4 Jahre alt, 14,1 kg schwer; Rachitis.

Status praesens: Dickes aufgeschwemmtes Kind, Kopf ausserordentlich dick, viereckig, die Gegend der Fontanelle höckrig. Thorax weich, stark ausgebildeter rachitischer Rosenkranz. Die Epiphysen der langen Röhrenknochen stark aufgetrieben, Knochen biegsam. Reconvalescent einer Bronchitis febrilis.

Nach 14 tägiger Ernährung, genau in derselben Weise wie in Versuch I, wurde der Urin täglich aufgefangen. Kurz vor Beginn der Kalkbestimmungen erhielt das Kind täglich drei Theelöffel Phosphorleberthran, welches auch während derselben, wie aus der Tabelle ersichtlich, zunächst fortgesetzt wurde, bis die eigenthumliche Versuchsreihe zum Weglassen desselben veranlasste.

Die gefundenen Werthe sind folgende:

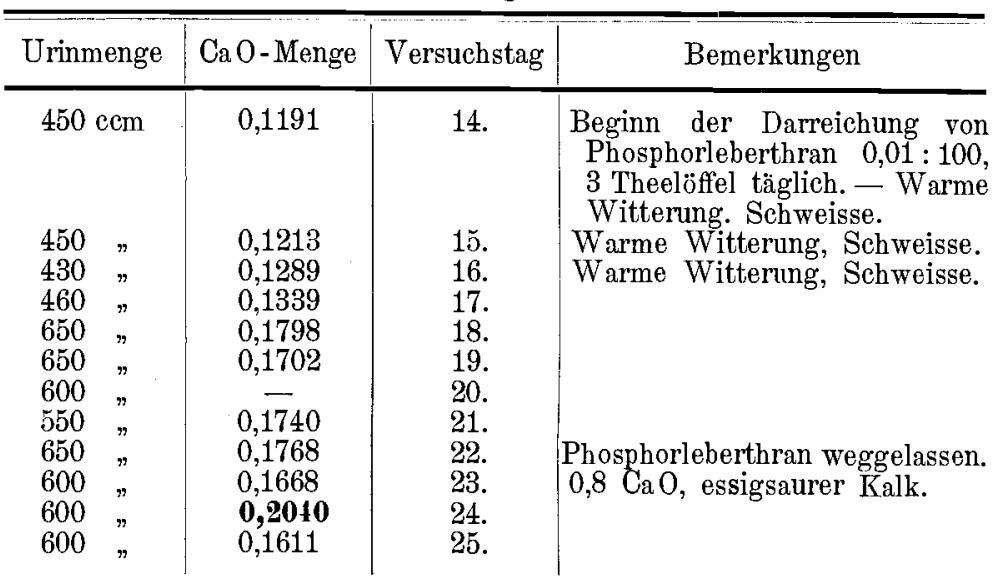

Auffällig ist die Inconstanz der Reihe und das stetige Anwachsen der ausgeschiedenen Kalkmengen. Wie spätere Versuche zeigten, beruht dieses darauf, dass das Kind kurz vor Beginn der Kalkbestimmungen und während der Dauer derselben bis zum 22. Tage Phosphorleberthran erhielt. Trotz dieses störenden Einflusses des Phosphorleberthrans ist aber auch hier die Wirkung der einmaligen Kalkgabe per os klar erkennbar. Der Harnkalk steigt sofort von 0,1668 auf 0,2040 , am folgenden, dem 25 . Versuchstage, kehrt die Ausscheidung wieder auf die frühere Höhe, 0,1611, zurück. Also auch hier bestätigte sich, dass eine einmalige Kalkzugabe per os den Harnkalk sofort steigert.

Es wurde nun weiterhin zunächst an diesem Patienten versucht, genaueres über die Wirkung des Phosphorleberthrans auf die Harnkalkausscheidung zu erfahren. Zunächst wurde das Medicament selbst auf Kalk untersucht.

Von $200 \mathrm{ccm}$, welche abdestillirt und deren Rackstand verdampft und verascht wurde, blieb so wenig Asche, dass eine Kalkbestimmung darin unmöglich erschien. Eine auch nur entfernt in Betracht kommende Kalkmenge konnte also nicht darin enthalten sein.

Fs wurde dann, um eine Täuschung zu vermeiden, nachdem 14 Tage kein Phosphorleberthran gegeben war, derselbe wieder eingefuhrt und nach acht Tagen die Kalkmenge im 24 stündigen Urin bestimmt.

Die folgende Tabelle giebt die gefundenen Zahlen.

\begin{tabular}{c|c|c}
\hline \hline Urinmenge & Ca O-Menge & Versuchstag \\
\hline $720 \mathrm{ccm}$ & 0,2084 & 9. \\
$700 "$ & 0,2239 & 10.
\end{tabular}


Am neunten Tage also ist die Kalkausscheidung im Urin bereits auf eine grössere Zahl gestiegen, als fruher durch Kalkverabreichung erreicht wurde.

Es wurde nun wieder mit dem Medicament abgebrochen; im Anschluss hieran sank die Kalkmenge im Harn nach einer Reihe von Tagen bis auf 0,1247 .

Diese Ergebnisse sind dazu angethan, einen steigernden Einfluss des Phosphorleberthrans auf die Kalkresorption bei gleichbleibendem Nahrungskalk im höchsten Grade wahrscheinlich zu machen, und zwar würde danach eine in gewissen Grenzen progradirte Steigerung der Kalkresorption während der Darreichung zu vermuthen sein. Die späteren, unten folgenden Beobachtungen an diesem Fall und der unten folgende Versuch III sind geeignet, diese Vermuthungen zu stützen. Etwas weiteres als Vermuthungen kann aber natürlich auf diesen vereinzelten Versuchen nicht basirt werden.

Es lag nun weiterhin nahe, zu prüfen, welcher der beiden Bestandtheile des Phosphorleberthrans den Einfluss auf die Kalkausscheidung zeigte. Daher wurde dem Kinde nach der erwähnten Pause ohne Medication, wobei die Kalkmenge bis auf 0,1247 herabsank, acht Tage lang einfacher gereinigter Leberthran gegeben, und dann unter Weitergabe desselben Leberthrans einige Tage die tägliche Kalkausscheidung bestimmt.

Die Werthe an den einzelnen Tagen finden sich in folgender Tabelle.

\begin{tabular}{c|c|c|c|c|c}
\hline \hline Urinmenge & CaO-Menge & Versuchstag & Urinmenge & CaO-Menge & Versuchstag \\
\hline $350 \mathrm{ccm}$ & 0,1472 & 9. & - & - & 12. \\
$580 \%$ & 0,1617 & 10. & $650 \mathrm{ccm}$ & 0,1621 & 13. \\
530, & 0,1641 & 11. & & &
\end{tabular}

Auch hier findet sich also ein Ansteigen der Kalkmenge im Urin, jedoch bei weitem nicht in dem Maasse wie bei Phosphorleberthran. Um nun den Phosphor fur sich zu prufen, wurde am 13. Tage der Leberthran weggelassen und nach zehntägiger Pause Phosphor in Emulsion in der gleichen Menge gegeben. Die Reihe war folgende:

\begin{tabular}{|c|c|c|c|c|c|}
\hline Urinmenge & CaO-Menge & Versuchstag & Urinmenge & CaO-Menge & Versuchstag \\
\hline $600 \mathrm{ccm}$ & 0,1524 & 14. & $550 \mathrm{ccm}$ & - & \\
\hline $600 \mathrm{ccm}$ & $0, \overline{1435}$ & 16. & 600 & 0,11 : & 24. \\
\hline 680 & & 17. & 680 & & \\
\hline 500 & 0,1228 & 18. & $650 "$ & 0,1103 & 26. \\
\hline $550 \mathrm{ccm}$ & 0,1314 & 20 . & $600 \mathrm{ccm}$ & 0,1223 & \\
\hline & & & 600 & & 29 . \\
\hline $600 \mathrm{ccm}$ & 0,1225 & 22. & 600 & 0,1215 & 30. \\
\hline
\end{tabular}

Der Versuch ergab somit, dass die Darreichung von Phosphor den Harnkalk nicht vermehrte, dass vielmehr während der achttägigen Versuchsdauer eine geringe Verminderung desselben eintrat. Es wurde hierauf mit der Phosphordarreichung abgebrochen. In der folgenden medicamentfreien Zeit von acht Tagen sank bei stets gleicher Ernährung (wie oben) und ohne sonstige dazwischentretende Umstände die Menge des vierundzwanzigstündigen Harnkalks noch etwas ab; sie erreichte den Werth von 0,08112. Dabei fing der Knabe an herumzulaufen, und die Festigkeit der Knochen schien etwas zugenommen zu haben.

Das Ergebniss dieser Untersuchungen (Versuch I und II) war einmal eine Bestätigung der Angaben Rüdel's über die Zunahme des Harnkalks im Anschluss an Kalkzugabe in der Nahrung; ferner aber fand sich, dass im Anschluss an die Verordnug von Phosphorleberthran $(0,01: 100,0,3$ Theelöffel täglich, = circa $12 \mathrm{~g}$ Leberthran, 0,0012 g Phosphor täglich) die Harnkalkmenge in auffälliger Weise anstieg, während sie dies nach der Anwendung der gleichen Menge Leberthran nur in geringem Maasse, nach der Verabreichung von täglich 0,0012 Phosphor aber gar nicht that. Diese Resultate waren so merkwürdig, dass eine Wiederholung der Versuche zunächst an demselben Patienten wünschenswerth erchien; da die bisherigen Vornahmen ihm nicht im mindesten geschadet hatten, vielmehr während derselben die Krankheit erheblich zurückgegangen war, so wurde beschlossen, die Darreichung von Phosphorleberthran zu wiederholen.

Versuch III. Demselben Patienten wurden täglich 3 mal 4 gleich $12 \mathrm{~g}$ Phosphorleberthran $(0,01: 100,0)$ einverleibt, bei fortdauernd gleicher Ernährung. Vom fünften Tage der Darreichung an wurde der 24 stündige Harnkalk bestimmt. In den Versuch wurde einmal die Darreichung eines Abfuhrmittels zur Prüfung der Wirkung des Durchfalls auf die Harnkalkmenge, sodann einmal eine Kalkzugabe eingeschoben. Folgendes war das Ergebniss:

\begin{tabular}{c|l|l}
\hline Urinmenge & \multicolumn{1}{|c|}{$\mathrm{CaO}$} & \multicolumn{1}{|c}{ Versuchstag } \\
\hline $550 \mathrm{ccm}$ & 0,08112 & 5. der Darreichung. \\
$520 "$ & 0,1026 & 6. von Phosphorleberthran. \\
$510 "$ & 0,0855 & 7. \\
$500 "$ & 0,1325 & 8. \\
$510 "$ & 0,1537 & 9. \\
$520 "$ & 0,2158 & 10.2 Löffel Oleum Ricini. \\
$400 "$ & $\mathbf{0 , 1 1 9 7}$ & 11. \\
$410 "$ & $\mathbf{0 , 1 2 6 9}$ & 12. \\
$500 "$ & 0,1869 & 13. \\
$510 "$ & 0,1820 & 14. \\
$560 "$ & 0,1789 & 15. \\
$550 "$ & 0,1703 & $16.0,8$ CaO als essigs. Kalk. \\
$6500 "$ & $\mathbf{0 , 2 3 1 5}$ & 17. \\
580 & 0,1545 & 18. Phosphorleberthran ab. \\
$500 "$ & 0,1143 & 28. \\
\hline
\end{tabular}

Wiederum trat ein rasches Ansteigen des Harnkalks auf; das eingeschobene Ricinusől bewirkte vorubergehenden Abfall, die eingeschlossene Kalkzugabe bewirkte vorubergehendes noch erheblicheres Steigen des Harnkalks. Nachdem dann am 18. Tag der Phosphorleberthran weggelassen war, fanden sich am 28. und 30. Tage wieder die Harnkalkziffern wie zu Beginn der Darreichung dieses Medicaments.

Hierdurch wurde immer wahrscheinlicher, dass es sich bei der beobachteten Wirkung des Phosphorleberthrans auf den Harnkalk um etwas gesetzmässiges handele, so sehr damit auch im Widerspruch stand, dass weder der Leberthran an sich, noch der Phosphor für sich allein dieselbe Wirkung entfaltete.

Die hier aufgeführten Versuche II und III sind an einem Kinde angestellt, dessen Kalkstoffwechsel sich an und für sich in verhältnissmässig grösseren Zahlen bewegte, bei welchen die Ausschläge einer Aenderung in deinselben dann auch um so deutlicher hervortraten. Der folgende Velsuch IV wurde bei einem Kinde mit geringerem Kalkstoffwechsel angestellt; die Ausschläge sind dementsprechend absolut genommen geringer, procentualiter jedoch dieselben wie im vorigen Versuch.

Versuch IV. Knabe, $1 / 2 \mathrm{~J}$ ahre, $10,4 \mathrm{~kg}$ schwer. Status praesens: mittelkräftiges Kind. Fontanelle noch nicht ganz geschlossen. Die Knochenränder derselben aufgetrieben. Rein rachitischer Rosenkranz, mittelstarke Epiphysenauftreibungen an den langen Rőhrenknochen.

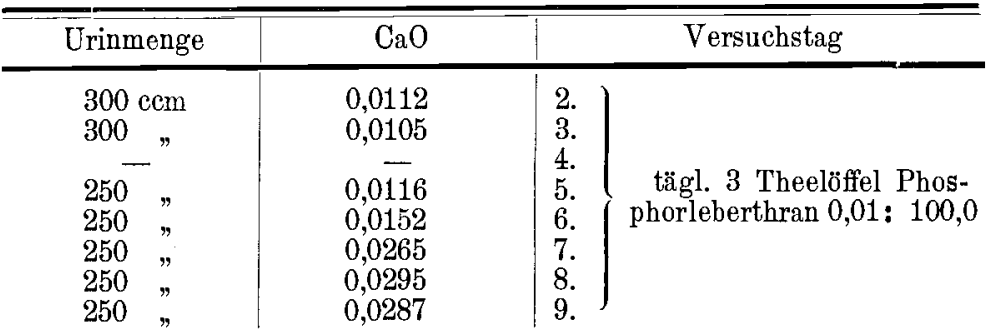

Nach 9 tägigem Verabreichen von Phosphorleberthran trat auch hier eine Steigerung der täglichen Kalkausscheidung um das doppelte der Normalmenge ein.

Nur beiläufig und anhangsweise gestatte ich mir, hier über einige Untersuchungen zu berichten, welche den Kalkgehalt des Harns einer osteomalacischen Wöchnerin, verglichen mit demjenigen normaler Wöchnerinnen betrafen.

Veranlassung $\mathrm{zu}$ diesen Untersuchungen gab ein Fall von Osteomalacie, der, während ich gerade mit den obigen Versuchen beschäftigt war, in der hiesigen Frauenklinik zur Beobachtung kam.

Frau C. A., 28 Jahre alt, III para, aus Reichenbach.

I. Geburt war eine spontane; Schädellage; Kind lebt.

II. Geburt: Steisslage, Kind starb intra partum.

III Geburt 5. Juni 1894, künstlich eingeleitet in der 34. Schwangerschaftswoche. Steisslage, Extraction, Kind lebt.

Die osteomalacischen Beschwerden begannen vom sechsten Monat der ersten Schwangerschaft, hörten vier Wochen post partum auf, begannen wieder im fiinften Monat der zweiten Schwangerschaft und schwanden wieder zwei Monate post partum. In der dritten Gravidität erschienen sie im dritten Monat; vom sechsten Monat an konnte Patientin nur mit Storcken gehen und lag meist zu Bett. Später ging sie nur am Tisch hin.

Befund: untere Rippen den Darmbeinschaufeln stark genähert; Thorax und Becken äusserst druckschmerzhaft, Schoossbogen wenig verengt; Promontorium leicht erreichbar, nach vorn verlagert, Conjugata diagonalis 10,75. Heftige Schmerzen in den Beinen und Hüften, namentlich bei Gehen und Stehen.

Bei Entlassug, 13 Tage post partum, wenig Besserung; Angaben uber späteres Befinden fehlen.

Die Kost der Wöchnerin war eine annähernd gleichmässige, in den ersten Tagen ublicherweise sehr einfache, vom sechsten Tage ab kräftigere. In den beiden ersten Tagen war die dem Urin beigemischte Blutmenge ziemlich gross; wiewohl dieselbe einen erheblichen Fehler kaum verursacht haben würde, stand ich doch von einer Untersuchung ab. Vom dritten Tage an aber wurde der tägliche Urin, so wie er bei spontaner Entleerung 
abfloss, gesammelt und in der oben angegebenen. Weise untersucht. Dabei wurde auch hier einmal eine Dosis anorganischen Kalks per os eingefuhrt. Ich bemerke, dass die Wöchnerin selbstverständlich ihr Kind nicht nährte.

Das Ergebniss der Untersuchungen war tolgendes:

Versuch V: Osteomalacische Wöchnerin.

\begin{tabular}{|c|c|c|}
\hline Urinmengen & $\mathrm{CaO}$ & Versuchstag \\
\hline $1400 \mathrm{ccm}$ & 0,5404 & 3. post partum \\
\hline 1430 & - & 4. $", \quad "$ \\
\hline 1500 & 0,3120 & 5.,$\quad "$ \\
\hline - & - & 6., \\
\hline $1250 "$ & 0,2100 & 7. $" \quad "$ \\
\hline $1300 "$ & 0,1818 & 8. $0,8 \mathrm{CaO}$ als essigsaurer Kalk \\
\hline 1250 & 0,2143 & 9. \\
\hline 1200 & 0,1171 & 10. \\
\hline$\overline{1210}$ & $0, \overline{0613}$ & 12. \\
\hline
\end{tabular}

Die Tabelle zeigt, dass der anfangs recht erhebliche Kalkgehalt des Urins im Laufe der ersten Untersuchungstage bedeutend und in grossen Sprüngen abnahm. Es zeigte sich dann ferner auch hier eine geringe vorübergehende Steigerung des Harnkalks auf einmalige Zugabe von anorganischem Kalk per os.

Zum Vergleich wurde auch an jeder normalen, ihre Kinder ebenfalls nicht stillenden Wöchnerin der Harnkalk bestimmt. Die Ergebnisse sind folgende:

Versuch VI: Normales Wochenbett nach normaler Geburt bei einer Gesunden; Kind lebend, reif.

\begin{tabular}{l|c|c}
\hline \hline Urinmenge & $\mathrm{CaO}$ & Tag des Wochenbetts \\
\hline $1580 \mathrm{ccm}$ & 0,4251 & 4. \\
$1450 "$ & 0,3701 & 5. \\
$1400 "$ & 0,3461 & 6. \\
$1520 "$ & 0,3696 & 7. \\
$1250 "$ & 0,3053 & 8. \\
$1050 "$ & 0,3549 & 9. \\
$1300 "$ & 0,3640 & 10.
\end{tabular}

Versuch VII: Normales Wochenbett nach normaler Geburt bei einer Gesunden; Kind lebend, reif.

\begin{tabular}{l|c|c}
\hline \hline Urinmenge & $\mathrm{CaO}$ & Tag des Wochenbetts \\
\hline $1500 \mathrm{ccm}$ & 0,1683 & 3. \\
$1400 "$ & 0,1620 & 4. \\
$1450 "$ & 0,1454 & 5. \\
$1170 \%$ & 0,1523 & 6. \\
$1050 "$ & 0,1199 & 7. \\
$1230 "$ & 0,1374 & 8.
\end{tabular}

Ein Vergleich dieser beiden Tabellen mit derjenigen von Versuch V (osteomalacische Wöchnerin) lässt nun allerdings einen bemerkenswerthen Unterschied erkennen. Die Abnahme des anfangs hohen Kalkgehalts ist in derselben Zeit bei der Osteomalacischen ausserordentlich viel stärker als bei den beiden normalen Frauen, bei welchen er nur wenig, übrigens in einem bei beiden merkwürdig gleichen Verhältniss, sinkt.

In Zahlen ausgedrückt ist das Verhältniss des Harnkalks am circa dritten zu dem am achten Wochenbetttag folgendes:

$$
\begin{aligned}
\text { bei der ersten Normalen } & =\text { ca. } 7: 6 \\
" \quad \text { zweiten } & =7: 6 \\
& =18: 6
\end{aligned}
$$

und hier sinkt es" in den folgenden vier Tagen bis auf das Verhältniss $10: 1$.

Es liegt natürlich nahe, das rasche Sinken der Kalkziffer bei der osteomalacischen Frau als ein Symptom der Kalkretention im Organismus anzusehen und aus demselben auf eine Apposition von Kalk in dem an Kalk verarmten Knochengerüst, d. h. auf beginnende Heilung der Krankheit zu schliessen. Das würde eine gewisse Bekräftigung dadurch erfahren, dass in der That auch die groben klinischen Erscheinungen der Osteomalacie bei der Kranken in der Folge rasch zurückgingen, wie sie das ja bekanntlich nach Beendigung der Schwangerschaft oft thun. Immerhin kann das nur eine vage Vermuthung sein, da unsere Kenntnisse vom Kalkstoffwechsel bei Osteomalacie und vom Verhalten des Harnkalks bei Heilung der Krankheit gleich Null sind.

Immerhin scheint mir diese Beobachtung wenigstens den Werth zu haben, dass sie zu neuen Versuchen auffordert.

Die Ergebnisse meiner Untersuchungen sind folgende:

1. Es hat sich bestätigt, dass bei der Rachitis eine Störung der Kalkresorption vom Darmcanal aus nicht besteht, dass mithin eine verminderte Kalkresorption nicht die Ursache der Krankheit sein kann.

2. Darreichung von Phosphorleberthran 0,01:100,0, drei Theelöffel täglich, steigert den Kalkgehalt des Urins erheblich und scheint demnach die Kalkresorption vom Darmcanal aus zu begünstigen. Gereinigter Leberthran an sich zeigte diese Wirkung in viel geringerem Maasse, Phosphor allein in den entsprechenden Dosen that es merkwürdigerweise fast gar nicht.

3. Bei einer osteomalacischen Wöchnerin im Wochenbett fand sich ein starkes Heruntergehen der (anfangs hohen) Urinkalkziffer, bis auf ein Zehntel am zwölften Tage, während bei zwei gesundel Wöchnerinnen der Urinkalk nur unerheblich herunterging. Möglicherweise hängt die erstere Erscheinung mit den Heilungsvorgängen der Ostomalacie zusammen. 